\title{
A Robust Sparse-Modeling Framework for Estimating Schizophrenia Biomarkers from fMRI
}

\author{
Keith Dillon ${ }^{\mathrm{a}, \mathrm{b}, *}$, Vince Calhoun ${ }^{\mathrm{c}, \mathrm{d}}$, Yu-Ping Wang ${ }^{\mathrm{a}, \mathrm{b}}$ \\ ${ }^{a}$ Department of Biomedical Engineering, Tulane University, New Orleans, LA, USA \\ ${ }^{b}$ Department of Global Biostatistics and Data Science, Tulane University, New Orleans, LA, USA \\ ${ }^{c}$ The Mind Research Network \& LBERI, Albuquerque, NM, USA \\ ${ }^{d}$ Department of Electrical Engineering, University of New Mexico, New Mexico, USA
}

\begin{abstract}
Background: Our goal is to identify the brain regions most relevant to mental illness using neuroimaging. State of the art machine learning methods commonly suffer from repeatability difficulties in this application, particularly when using large and heterogeneous populations for samples.
\end{abstract}

New Method: We revisit both dimensionality reduction and sparse modeling, and recast them in a common optimization-based framework. This allows us to combine the benefits of both types of methods in an approach which we call unambiguous components. We use this to estimate the image component with a constrained variability, which is best correlated with the unknown disease mechanism.

Results: We apply the method to the estimation of neuroimaging biomarkers for schizophrenia, using task fMRI data from a large multi-site study. The proposed approach yields an improvement in both robustness of the estimate and classification accuracy.

Comparison with Existing Methods: We find that unambiguous components incorporate roughly two thirds of the same brain regions as sparsity-based methods LASSO and elastic net, while roughly one third of the selected regions differ. Further, unambiguous components achieve superior classification accuracy in differentiating cases from controls.

Conclusions: Unambiguous components provide a robust way to estimate important regions of imaging data.

Keywords: Schizophrenia, Functional MRI, Sparsity, PCA, Optimization 


\section{Introduction}

In this paper our goal is to find the most relevant brain regions given labeled neuroimaging data; the ultimate goal is to use those results to understand disease mechanisms, as well as to provide biomarkers to help diagnose (i.e., classify) patients as having disease or not. There is a significant need for techniques which can robustly extract information in such a problem. Neuroimaging, particularly functional neuroimaging, has provided a wealth of intriguing information regarding brain function, but has yet to show clear value to psychiatric diagnosis [19]. Despite this, impressive results have been achieved with machine learning techniques such as support vector machines, which demonstrate high classification accuracies [24]. Reproducibility problems persist however [5], with an apparent trend towards poorer performance for larger studies [27].

The identification of meaningful components of the data is a key benefit of many feature selection techniques [17], in addition to providing improvements in performance of subsequent classification stages [9]. In a typical neuroimaging study there may be tens or hundreds of subjects, each with an image consisting of up to hundreds of thousands of voxels, resulting in an extremely underdetermined problem. A popular category of feature selection approaches is regularized regression techniques such as LASSO [29] and related methods employing sparse models $[6,21]$. Such supervised techniques impose task-specific information (the data labels), with a penalty term to incorporate prior knowledge. In the case of LASSO, the prior knowledge amounts to a presumption of sparsity on the relationship between image data and labels, i.e., that the underlying biological mechanism involves a limited number of the imaged voxels. Unfortunately, if the problem is both very underdetermined and very noisy, then the regularized solution may not be a particularly superior choice; many solutions may potentially be of similar or even equal probability to each other. For example in the underdetermined case, the LASSO solution may not be unique for highly-structured datasets $[30,33]$. Along these lines, we simply may not have sufficient confidence in the

\footnotetext{
*Corresponding author. Tel. 1-504-988-1547

Email address: kdillon1@tulane.edu (Keith Dillon)
} 
validity of our prior knowledge formulation to presume that the most regular solution is preferable to those even moderately as regular.

From a different direction, dimensionality reduction techniques [20] offer a more robust approach to feature selection in neuroimaging data. An example is principal component analysis (PCA) [13], which finds basis vectors for the space containing the data variation. This set of basis vectors can be viewed as robust in the sense that they are common to all solutions of any linear regression based on the data. In statistics a closely-related concept is estimable functions [23]. We will refer to components with such a property as unambiguous, and examine this more formally in the next section. Of course such components only describe the data itself, not necessarily the aspects of the data pertinent to our application, such as for finding information most related to a disease phenotype. A common approach is to utilize PCA and related factoring methods in a supervised fashion by choosing a subset of factors which best correlate with the labels. Supervised factoring techniques such as the "Supervised PCA" of [3], and related methods, can be viewed as a more sophisticated version of this technique, finding a transformation of the data such that the correlation with the labels is maximized. However these techniques are not able to incorporate prior knowledge, such as sparsity of the mechanism, into this transformation. Techniques have been developed which do incorporate sparsity into unsupervised factoring techniques (e.g., sparse PCA [35]) in a heuristic sense, though this differs from presuming sparsity of the underlying mechanism; the presumption of sparsity is applied to the structure of the component itself rather than to the unknown mechanism. Hybrid methods have been proposed which perform PCA following a pre-screening step which picks a subset of variables over a correlation threshold [2] or in known pathways [22]. However we would prefer to incorporate multi-variable relationships in the screening component.

In this paper, we develop an approach which combines the benefits of both regularized estimates and dimensionality reduction by simultaneously enforcing unambiguity and prior knowledge in calculating components. We start by reviewing dimensionality reduction from the perspective of unambiguous components. Then we review related regularization methods and show how they motivate our approach to incorporate prior knowledge into unambiguous 
components. By maximizing the correlation with the mechanism, we calculate components which identify the most important regions in the data. We use a simulation to show how this component performs robustly in the face of inaccurate prior knowledge, by demonstrating that the correlation still remains controlled as the prior knowledge is relaxed. Finally, we show a successful application to biomarker identification where we identify features of fMRI data which relate to schizophrenia more accurately than other methods which utilize sparsity as prior knowledge.

\section{Materials and Methods}

We will consider the linear model $\mathbf{A x}=\mathbf{b}+\mathbf{n}$ where $\mathbf{A}$ is a $m \times n$ data matrix with $n>m$, containing samples as rows, and variables as columns; $\mathbf{b}$ is the phenotype encoded into a vector of labels such as case or control; the solution $\mathbf{x}$ is the unknown model parameters that relate $\mathbf{A}$ to $\mathbf{b}$; and $\mathbf{n}$ is a noise vector about which we have only statistical information. We will also assume the means have been removed from $\mathbf{b}$ and the columns of $\mathbf{A}$ to simplify the presentation. The rows of $\mathbf{A}$ are provided by the contrast images from individual study subjects, so a predictor $\mathbf{x}$ selects a weighted combination of voxels (i.e., columns of $\mathbf{A}$ ) which relates the imagery to the case-control status. By examining the weightings in this combination we hope to learn more about the spatial distribution of causes or effects of the disease, which we will term the "mechanism" in this paper. The model is depicted in Fig. 1 (a), where we depict the true solution $\mathbf{x}$ as the mechanism whereby brain activity relates to the measured phenotypes. Of course there are far more unknown variables than samples, hence our linear system is underdetermined and there will be many possible $\mathbf{x}$ which solve the system. One way to address this problem is to impose prior knowledge about the biological mechanism, such as a preference for sparser $\mathbf{x}$, and select the solution which best fulfills this preference. We will review this approach in a later section. Another approach is to restrict our analysis to components of the solution which may be more easily estimated, such as via dimensionality reduction; an intuitive example of this approach is to group voxels into low-resolution regions. These alternatives are depicted in Fig. 1 (b), as extremes on a continuum of possible methods, where the goal of this paper is to find 
(a)

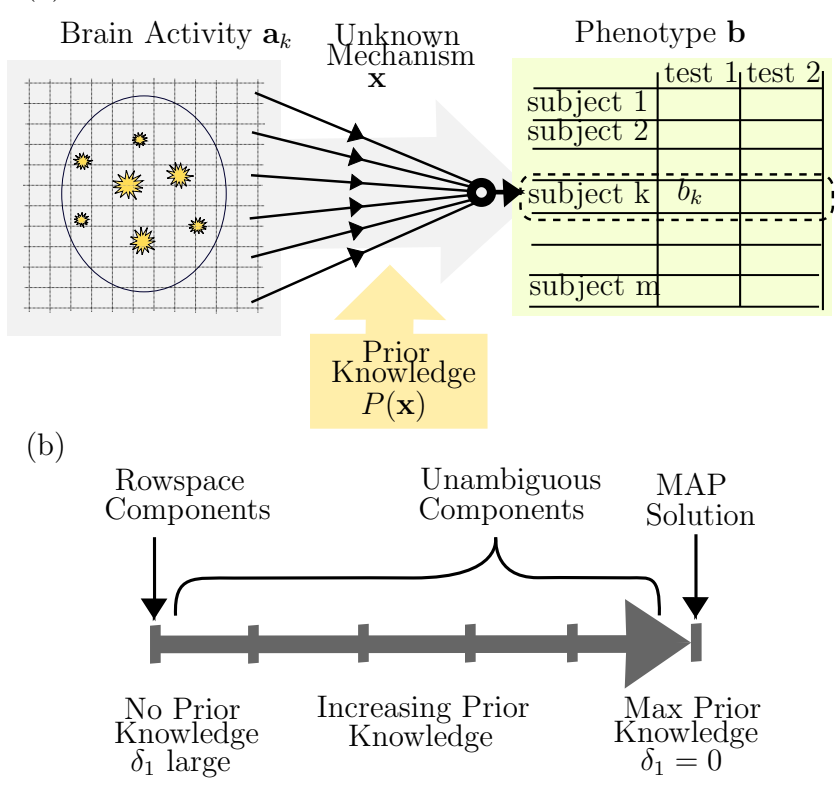

Figure 1: (a) Mathematical model $\mathbf{A x}=\mathbf{b}$, where $\mathbf{x}$ describes the mechanism that relates brain activity to phenotype (psychiatric assessments). The contrast map for a single subject, $\mathbf{a}_{k}$, provides the $k t h$ row of A. As there are still many unknown biological variables, the problem is underdetermined and $\mathbf{x}$ cannot be found uniquely; instead we must settle for a probable result such as a maximum likelihood solution which utilizes prior knowledge, or an estimable component of $\mathbf{x}$. (b) Continuum between rowspace components and most probable solution, based on increasing confidence in the prior knowledge, which we control by the relaxation parameter $\delta_{1}$.

intermediate information which utilizes the benefits of both extremes.

\subsection{Dimensionality Reduction}

To see how dimensionality reduction can apply to the estimation of mechanism, consider the Singular Value Decomposition (SVD) of $\mathbf{A}=\mathbf{U S V}^{T}$, where $\mathbf{S}$ is a diagonal matrix of singular values $\sigma_{i}$, and $\mathbf{U}$ and $\mathbf{V}$ contain the left and right singular vectors $\mathbf{u}_{i}$ and $\mathbf{v}_{i}$. In Principal Components Analysis (PCA) the focus is on this expansion itself, however our focus here is on the mechanism which we model by $\mathbf{x}$. If we plug $\mathbf{U S V}^{T}$ into $\mathbf{A x}=\mathbf{b}$ and apply the transformation $\mathbf{U}^{T}$ to both sides, we get a diagonalized system with decoupled equations of the form $\sigma_{i} \mathbf{v}_{i}^{T} \mathbf{x}=\mathbf{u}_{i}^{T} \mathbf{b}$. This equation tell us that, while we can't calculate the true $\mathbf{x}$ in the underdetermined case, we can calculate components of $\mathbf{x}$ corresponding to nonzero 
singular values. One way to view this property is by considering that these components are constant for all possible $\mathbf{x}$ given our linear system. In other words, $\sigma_{i} \mathbf{v}_{i}^{T} \mathbf{x}$ calculates the same value for any solution in the set $\{\mathbf{x} \mid \mathbf{A x}=\mathbf{b}\}$. In terms of our application, this means while we can't identify the true mechanism $\mathbf{x}$, we can extract reliable components of it. For example, we might be able to coarsely identify large regions containing important activity, without being able to pinpoint particular voxels within those regions. Of course the SVD selects vectors based on orthogonal directions of ranked data variation, which may not be best suited to provide information about the disease mechanism. We will address this later by optimizing the choice of component, but first we will formally consider this property of constant components so that we may extend it later to incorporate prior knowledge.

\subsection{Unambiguous Components without Prior Knowledge}

In this section we will introduce the idea of unambiguous components in the noise-free case without prior knowledge. The concept is demonstrated geometrically in Fig. 2 (a), where the useful information in $\mathbf{A}$ forms the matrix rowspace; this contains the dimensions over which we have a diversity of data which we can compare to the phenotype b. Dimensions perpendicular to the rowspace form the nullspace, directions over which our data does not vary. For example if our dataset was composed of subjects with the same age, then we cannot perform a regression to see how disease risk relates to age. In terms of components of the data, if $\mathbf{c}$ is a loading vector and $\mathbf{a}_{i}$ is a sample (row of $\mathbf{A}$ ), then $\mathbf{c}^{T} \mathbf{a}_{i}$ is potentially useful information when $\mathbf{c}$ is in the rowspace, and useless information (in fact always zero) when $\mathbf{c}$ is in the nullspace. An equivalent perspective, depicted in Fig. 2 (b), is the geometry of the solution set to the regression model. This is the affine set $S_{E C}$, composed of an offset (the least-length solution $\mathbf{x}_{0}$ ) plus a vector from the nullspace.

$$
S_{E C}=\{\mathbf{x} \mid \mathbf{A} \mathbf{x}=\mathbf{b}\} .
$$

The subscript " $E C$ " refers to $\mathbf{x}$ being only equality-constrained (i.e., we have imposed no prior knowledge yet). The rowspace vectors yield estimable components of $\mathbf{x}$ given the data, while nullspace vectors give the dimensions of ambiguity we have about $\mathbf{x}$. We will formulate this relationship rigorously next. 
(a)

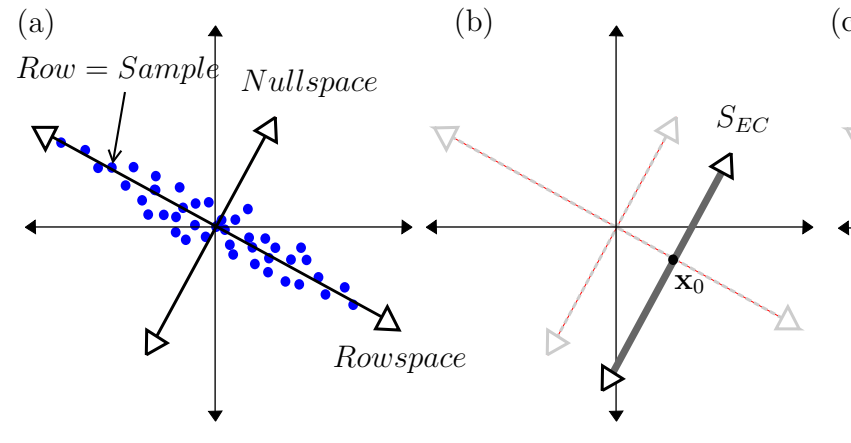

(c)

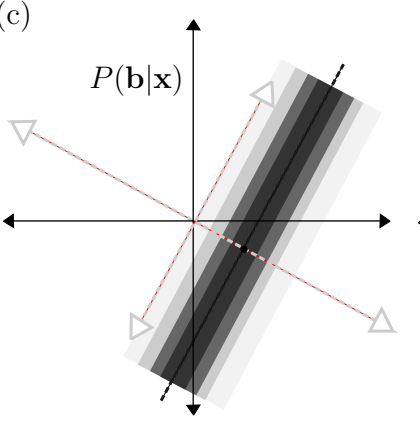

(d)

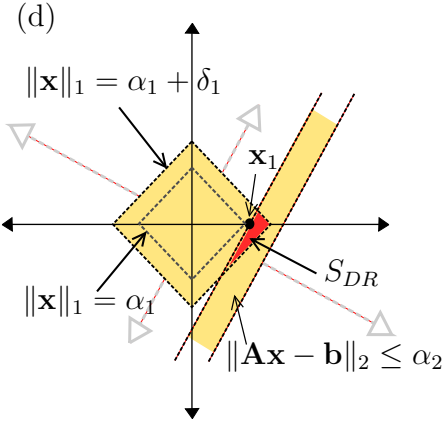

Figure 2: Two-dimensional example: (a) The rowspace of $\mathbf{A}$ is the direction of data diversity, while the nullspace is perpendicular directions, which lack diversity. (b) The solution set to $\mathbf{A x}=\mathbf{b}$ is the affine space $S_{E C}$, which is parallel to the nullspace; the ambiguity due to lack of data diversity results in ambiguity of possible solutions forming $S_{E C}$. (c) The distribution of $\mathbf{x}$ resulting from the likelihood $P(\mathbf{b} \mid \mathbf{x})$, the high probability region is nearest $S_{E C}$. (d) The set $S_{D R}$ formed by the intersection of a bound on $P(\mathbf{b} \mid \mathbf{x})$ enforced by $\|\mathbf{A} \mathbf{x}-\mathbf{b}\|_{2} \leq \alpha_{2}$, and a bound on the prior distribution $P(\mathbf{x})$ enforced by $\|\mathbf{x}\|_{1} \leq \alpha_{1}$; the LASSO solution $\mathbf{x}_{1}$ is based on a trade-off where the combination of bounds must be as tight as possible.

Recall that the rowspace of $\mathbf{A}$ is defined as the set of all possible linear combinations of rows, i.e., $\mathcal{R}\left(\mathbf{A}^{T}\right)=\left\{\mathbf{c} \mid \mathbf{A}^{T} \mathbf{y}=\mathbf{c} \forall \mathbf{y} \in \mathbb{R}^{m}\right\}$. We can equivalently view this as a set whose members take on a constant value over $S_{E C}$. In other words, they form the set of unambiguous components for $S_{E C}$. We state this simple but important fact in Theorem 1 .

Theorem 1. The following two statements are equivalent:

1. $\mathbf{c} \in \mathcal{R}\left(\mathbf{A}^{T}\right)$.

2. $\mathbf{c}^{T} \mathbf{x}=\mu$ for all $\mathbf{x} \in S_{E C}$, where $\mu$ is a constant.

Proof. It is straightforward to note that, as $\mathbf{A}^{T} \mathbf{y}=\mathbf{c}$, we have $\mathbf{c}^{T} \mathbf{x}=\mathbf{y}^{T} \mathbf{A} \mathbf{x}=\mathbf{y}^{T} \mathbf{b} \equiv \mu$. However it will be useful to our subsequent extension to note the fact that the rowspace is the orthogonal complement to the nullspace, and can be also written as $\mathcal{R}\left(\mathbf{A}^{T}\right)=$ $\left\{\mathbf{c} \mid \mathbf{c}^{T} \mathbf{z}=0 \forall \mathbf{z} \in \mathcal{N}(\mathbf{A})\right\}$. Equivalently, any vector $\mathbf{z}$ in the nullspace may be described as a difference between solutions, i.e., $\mathbf{z}=\mathbf{x}_{1}-\mathbf{x}_{2}$, where $\mathbf{x}_{1}, \mathbf{x}_{2} \in S_{E C}$. Therefore the rowspace may also be written as $\mathcal{R}\left(\mathbf{A}^{T}\right)=\left\{\mathbf{c} \mid \mathbf{c}^{T} \mathbf{x}_{1}=\mathbf{c}^{T} \mathbf{x}_{2} \forall \mathbf{x}_{1}, \mathbf{x}_{2} \in S_{E C}\right\}$, which explicitly states the equivalence in Theorem 1.

The principal components of $\mathbf{A}$ corresponding to the $r$ nonzero singular values must 
be in the rowspace. Again, given a singular-value decomposition $\mathbf{A}=\mathbf{U S V}^{T}$, we have $\mathbf{V S U}^{T} \mathbf{y}=\mathbf{c}$ for $\mathbf{c} \in \mathcal{R}\left(\mathbf{A}^{T}\right)$, and hence $\mathbf{V}^{T} \mathbf{c}=\mathbf{S U}^{T} \mathbf{y}=\sum_{i=1}^{r} \sigma_{i} \mathbf{u}_{i}$, where the $\mathbf{u}_{i}$ for $i \in\{1, \ldots, r\}$ form a basis for the rowspace. So principal components are unambiguous components.

Later we will also consider how to optimize the choice of component such that it also computes a score which is maximally useful for an application (such as to identify important image regions for classification of disease). But first we will extend the concept of unambiguous components to incorporate prior knowledge. Our focus on components of the mechanism $\mathbf{x}$ rather than of the data $\mathbf{a}_{i}$ allows us to incorporate prior knowledge which applies to $\mathbf{x}$ into the method, such as the presumption of a sparse biological mechanism. In effect, we will utilize prior knowledge to further restrict the variation in $\mathbf{x}$ and potentially increase the estimable components of $\mathbf{x}$.

\subsection{LASSO and Elastic Net}

In this section we will review closely-related estimation methods which utilize prior knowledge, to provide an intuitive basis for our mathematical formulation. The Maximum A Posteriori (MAP) estimate $\mathbf{x}_{M A P}$ is the solution which maximizes the posterior probability $P(\mathbf{x} \mid \mathbf{b})$ over $\mathbf{x}$. Using Bayes' theorem, we can form the equivalent problem, $\arg \max _{\mathbf{x}} P(\mathbf{b} \mid \mathbf{x}) P(\mathbf{x})$, which utilizes the likelihood $P(\mathbf{b} \mid \mathbf{x})$ (essentially the distribution for $\mathbf{n}$ ), and the prior probability distribution $P(\mathbf{x})$. It is the form of this prior distribution which we refer to as the prior knowledge. A common approach is to take the log of this objective to get a penalized regression problem. With a Gaussian distribution for $\mathbf{n}$ and a Laplace distribution for $\mathbf{x}$, taking the log yields the most well-known version of LASSO, $\min _{\mathbf{x}}\|\mathbf{A x}-\mathbf{b}\|_{2}^{2}+\lambda\|\mathbf{x}\|_{1}$. LASSO was originally proposed in the form, $\min _{\mathbf{x}}\|\mathbf{A x}-\mathbf{b}\|_{2}^{2}$ subject to $\|\mathbf{x}\|_{1} \leq \alpha_{1}$, with the other version sometimes described as the Lagrangian form. It can be shown that for any $\lambda$, a $\alpha_{1}$ exists such that these optimization problems have the same minimizer, $\mathbf{x}_{1}$. We can similarly form the feasibility problem (an optimization problem which stops when any feasible point is found, hence we simply use a 
zero for the objective as it is irrelevant [4]),

$$
\begin{gathered}
\mathbf{x}_{1}=\quad \arg \min _{\mathbf{x}} 0 \\
\|\mathbf{x}\|_{1} \leq \alpha_{1} \\
\|\mathbf{A} \mathbf{x}-\mathbf{b}\|_{2} \leq \alpha_{2}
\end{gathered}
$$

In this paper we will focus on sets of the general form of this feasible set we will denote as $S_{D R}$. In terms of the statistical distributions, the constraints on the norms amount to constraints on the probabilities, as in

$$
\begin{aligned}
S_{D R} & =\left\{\mathbf{x} \mid\|\mathbf{x}\|_{1} \leq \alpha_{1},\|\mathbf{A} \mathbf{x}-\mathbf{b}\|_{2} \leq \alpha_{2}\right\} \\
& =\left\{\mathbf{x} \mid P(\mathbf{x}) \geq P_{\min }, P(\mathbf{A} \mathbf{x}-\mathbf{b}) \geq P_{\min }\right\}
\end{aligned}
$$

The subscript $D R$ refers to the combination of denoising and regularization constraints. It can also be shown that $\mathbf{x}_{1}$ which solves the previous versions, will provide a member of this set. Therefore, we may write the LASSO problem as

$$
\begin{array}{r}
\mathbf{x}_{1}=\quad \arg \min _{\mathbf{x}} 0 \\
\mathbf{x} \in S_{D R}
\end{array}
$$

The elastic net [34] can be viewed as a variation on LASSO, where (under the Bayesian framework) the Laplace prior distribution for the prior is replaced by a product of Laplace and Gaussian distributions. Taking the log of the posterior distribution leads to the wellknown problem $\min _{\mathbf{x}}\|\mathbf{A} \mathbf{x}-\mathbf{b}\|_{2}^{2}+\lambda_{1}\|\mathbf{x}\|_{1}+\lambda_{2}\|\mathbf{x}\|_{2}^{2}$. As with LASSO, we may achieve the same solution using different optimization problems. We wish to use the same constraints as earlier, so we form the related problem,

$$
\begin{aligned}
& \mathbf{x}_{2}=\quad \arg \min _{\mathbf{x}}\|\mathbf{x}\|_{2}^{2}=\arg \min _{\mathbf{x}}\|\mathbf{x}\|_{2}^{2} \\
& \|\mathbf{x}\|_{1} \leq \alpha_{1} \quad \mathbf{x} \in S_{D R} . \\
& \|\mathbf{A x}-\mathbf{b}\|_{2} \leq \alpha_{2}
\end{aligned}
$$

Recall that LASSO seeks any single solution within $S_{D R}$. In that case, $\alpha_{1}$ and $\alpha_{2}$ are chosen as small as possible so the set is (hopefully) a singleton. Elastic net, on the other hand, seeks 
the least length solution in $S_{D R}$, where we may choose $\alpha_{1}$ and $\alpha_{2}$ more loosely, to trade-off desirable properties of this solution. These are depicted in Fig. 2 (d), where the LASSO solution, $\mathbf{x}_{1}$ is formed by tightening the bounds on the two sets (one representing the prior and one representing the noise) to minimal intersection. The elastic net solution, by contrast, relaxes the constraints on the two sets forming the larger set $S_{D R}$, and selection of the least length solution in this intersection. Next we will extend the unambiguous component idea to utilize $S_{D R}$, thereby incorporating prior knowledge into the framework.

Finally, note that the norms $\|\cdot\|_{1}$ and $\|\cdot\|_{2}$ may be replaced with other choices of norms representing other forms of prior knowledge, such as the $\ell_{\infty}$-norm, which imposes hard limits on the unknowns or the error, and which might result from box constraints [1] or a minimax regression [26]. More complex forms of penalties are used in the elastic net [34], and methods utilizing mixed norms [18] such as group LASSO [32]. The methods and theoretical results in this paper are generally the same as long as the choice fulfills the mathematical properties of a norm (though this is not a strict requirement).

\subsection{Unambiguous Components Subject to Prior Knowledge}

Earlier, we introduced unambiguous components as components of the unknown solution, which computed a constant score over all possible solutions. Now we will extend this concept to replace the set of possible solutions $\left(S_{E C}\right)$ with a set of highly-probable solutions, for which we will use $S_{D R}$. We will also extend the requirement that the score be constant to a requirement that their variation is limited within a fixed bound. So in effect, given a pool of solutions, which we may view as the most likely hypotheses for the underlying mechanism, we will seek components which compute approximately-constant scores for every likely mechanism.

We incorporate prior knowledge as well as bounds on the score by relaxing the rowspace with the following generalization,

$$
\begin{aligned}
R_{S}(\epsilon) & =\left\{\mathbf{c}|| \mathbf{c}^{T} \mathbf{x}_{1}-\mathbf{c}^{T} \mathbf{x}_{2} \mid \leq \epsilon \forall \mathbf{x}_{1}, \mathbf{x}_{2} \in S_{D R}\right\} \\
& =\left\{\mathbf{c} \mid \mathbf{c}^{T} \mathbf{x}_{1}-\mathbf{c}^{T} \mathbf{x}_{2} \leq \epsilon \forall \mathbf{x}_{1}, \mathbf{x}_{2} \in S_{D R}\right\}
\end{aligned}
$$


Here we have relaxed the ambiguity to potentially allow a positive bound $\epsilon$, as well as replaced $S_{E C}$ with $S_{D R}$. Note that we were able to remove the absolute value in Eq. (8) because membership in $R_{S}(\epsilon)$ requires the inequality hold for all $\mathbf{x}_{1}, \mathbf{x}_{2} \in S_{D R}$, hence if it holds for $\mathbf{x}_{1}^{\prime}, \mathbf{x}_{2}^{\prime} \in S_{D R}$, it must also hold for $\mathbf{x}_{1}^{\prime \prime}, \mathbf{x}_{2}^{\prime \prime}$ where $\mathbf{x}_{1}^{\prime \prime}=\mathbf{x}_{2}^{\prime}$ and $\mathbf{x}_{2}^{\prime \prime}=\mathbf{x}_{1}^{\prime}$. To highlight the view of this set as a generalization of the rowspace, we state the following simple generalization of Theorem 1, without proof,

Theorem 2. The following two statements are equivalent:

1. $\mathbf{c} \in R_{S}(\epsilon)$.

2. $\mu-\frac{1}{2} \epsilon \leq \mathbf{c}^{T} \mathbf{x} \leq \mu+\frac{1}{2} \epsilon$ for all $\mathbf{x} \in S_{D R}$, where $\mu$ is a constant.

Note that while this formulation appears quite simple, it will generally not be possible to describe $R_{S}(\epsilon)$ in a closed form or analytically determine whether a vector is a member of $R_{S}(\epsilon)$. However we may use convex optimization theory to formulate $R_{S}(\epsilon)$ as a system of inequalities. We defined $R_{S}(\epsilon)$ via the test $\mathbf{c}^{T} \mathbf{x}_{1}-\mathbf{c}^{T} \mathbf{x}_{2} \leq \epsilon$, which may also be written as $\mathbf{c}^{T}\left(\mathbf{x}_{1}-\mathbf{x}_{2}\right) \leq \epsilon$. We may use optimization to directly perform this test as follows,

$$
\begin{gathered}
p=\max _{\mathbf{x}, \mathbf{x}^{\prime}} \mathbf{c}^{T}\left(\mathbf{x}-\mathbf{x}^{\prime}\right) \\
\mathbf{x} \in S_{D R} \\
\mathbf{x}^{\prime} \in S_{D R} .
\end{gathered}
$$

If the optimal value $p \leq \epsilon$, then we were unable to find any pair of solutions to demonstrate an ambiguity in the component score greater than $\epsilon$, and hence $\mathbf{c} \in R_{S}(\epsilon)$. Similar methods have been proposed elsewhere to use optimization to test boundedness [10] and uniqueness $[11,30]$ of solutions to systems with particular forms of prior knowledge; in those cases the test was performed over individual variables, whereas we will use a test for a general component $\mathbf{c}$ here. $S_{D R}$ is a convex set so this is a straightforward convex optimization problem for either maximization or minimization, and so we can be assured of finding a global optima with an efficient algorithm [4]. If we replace $S_{D R}$ with $S_{E C}$ we have the 
equality-constrained linear program [14],

$$
\begin{gathered}
p=\max _{\mathbf{x}, \mathbf{x}^{\prime}} \mathbf{c}^{T}\left(\mathbf{x}-\mathbf{x}^{\prime}\right) \\
\mathbf{A} \mathbf{x}=\mathbf{b} \\
\mathbf{A} \mathbf{x}^{\prime}=\mathbf{b} .
\end{gathered}
$$

Unless $\mathbf{c}$ is in the rowspace of $\mathbf{A}$, this optimization problem will be unbounded. So the optimality condition for Eq. (10) is the now-familiar requirement that a solution can be found to $\mathbf{A}^{T} \mathbf{y}=\mathbf{c}$.

It is not useful to relax the ambiguity in the equality-constrained case (there, $p$ is either zero or infinite), but for $S_{D R}$ we can form general conditions for $\mathbf{c} \in R_{S}(\epsilon)$ incorporating a relaxed upper limit $p \leq \epsilon$. The analogous set of conditions achieved using $S_{D R}$ instead of $S_{E C}$, and allowing a non-zero $\epsilon$, is the following, as we will show with Theorem 3 ,

$$
\begin{gathered}
\mathbf{b}^{T}\left(\mathbf{y}+\mathbf{y}^{\prime}\right)+\alpha_{1}\left(\lambda_{1}+\lambda_{1}^{\prime}\right)+\alpha_{2}\left(\lambda_{2}+\lambda_{2}^{\prime}\right) \leq \epsilon \\
\left\|\mathbf{A}^{T} \mathbf{y}-\mathbf{c}\right\|_{\infty} \leq \lambda_{1} \\
\left\|\mathbf{A}^{T} \mathbf{y}^{\prime}+\mathbf{c}\right\|_{\infty} \leq \lambda_{1}^{\prime} \\
\|\mathbf{y}\|_{2} \leq \lambda_{2} \\
\left\|\mathbf{y}^{\prime}\right\|_{2} \leq \lambda_{2}^{\prime} .
\end{gathered}
$$

This is the generalization of the rowspace condition $\mathbf{A}^{T} \mathbf{y}=\mathbf{c}$ to $R_{S}(\epsilon)$. We show this with the following theorem,

Theorem 3. If there exists a $\mathbf{y}, \mathbf{y}^{\prime}, \lambda_{1}, \lambda_{1}^{\prime}, \lambda_{2}$ and $\lambda_{2}^{\prime}$ such that $\mathbf{c}$ is a solution to Eqs. (11), then $\mathbf{c} \in R_{S}(\epsilon)$.

Proof. We can test the limits of $\mathbf{c}^{T} \mathbf{x}$ with the optimization problem of Eq. (9) with $S=S_{D R}$

$$
\begin{gathered}
p=\max _{\mathbf{x}, \mathbf{x}^{\prime}} \mathbf{c}^{T}\left(\mathbf{x}-\mathbf{x}^{\prime}\right) \\
\|\mathbf{x}\|_{1} \leq \alpha_{1} \\
\left\|\mathbf{x}^{\prime}\right\|_{1} \leq \alpha_{1} \\
\|\mathbf{A x}-\mathbf{b}\|_{2} \leq \alpha_{2} \\
\left\|\mathbf{A} \mathbf{x}^{\prime}-\mathbf{b}\right\|_{2} \leq \alpha_{2} .
\end{gathered}
$$


By forming the dual [4] of the optimization problem in Eq. (12), we can get an upper bound on the optimal. The dual optimization problem is

$$
\begin{gathered}
d=\min _{\substack{\mathbf{y}, \mathbf{y}^{\prime}, \lambda_{1}, \lambda_{1}^{\prime}, \lambda, \lambda^{\prime}}}\left\{\mathbf{b}^{T}\left(\mathbf{y}+\mathbf{y}^{\prime}\right)+\alpha_{1}\left(\lambda_{1}+\lambda_{1}^{\prime}\right)+\alpha_{2}\left(\lambda+\lambda^{\prime}\right)\right\} \\
\left\|\mathbf{A}^{T} \mathbf{y}-\mathbf{c}\right\|_{1}^{*} \leq \lambda_{1} \\
\left\|\mathbf{A}^{T} \mathbf{y}^{\prime}+\mathbf{c}\right\|_{1}^{*} \leq \lambda_{1}^{\prime} \\
\|\mathbf{y}\|_{2}^{*} \leq \lambda_{2} \\
\left\|\mathbf{y}^{\prime}\right\|_{2}^{*} \leq \lambda_{2}^{\prime},
\end{gathered}
$$

where $\|\cdot\|^{*}$ is the dual norm of $\|\cdot\|$. For example the dual norm for the $\ell_{1}$-norm is the $\ell_{\infty^{-}}$ norm (we provide this general result to allow for other choices of norms if desired). The weak duality condition [4], which always holds, tells us that $p \leq d$. By constraining the objective of Eq. (13) to be bounded by $\epsilon$, which would mean $\mathbf{c} \in R_{S}(\epsilon)$, we get the conditions of Eqs. (11).

Note that strong duality holds for convex optimization problems as well as many nonconvex problems under a broad set of conditions, the most well-known being Slater's condition [4]. When strong duality holds, the converse of Theorem 3 holds as well, and so our conditions hold for all elements of $R_{S}(\epsilon)$. We will presume this is the case. Next we will consider the choice of useful components which fulfill the conditions of Eqs. (11).

\subsection{Optimizing Components}

The motivation and strategy of our approach are summarized in Fig. 3. For a hypothetical example, we give several potential solutions to $\mathbf{A x}=\mathbf{b}$ which we denote as $\mathbf{x}_{(i)}$, where $\mathbf{A x}_{(i)}=\mathbf{b}$ for all $i$. Recall each solution is a potential mechanism. For example, potential solution $\mathbf{x}_{(1)}$ suggests the mechanism is based on activity at voxel $k_{2}$, while potential solution $\mathbf{x}_{(4)}$ suggests the mechanism is based on activity at voxel $k_{1}$. Choosing any single solution such as the MAP solution is risky as it may lead us to the wrong voxel if incorrect. We can analyze the risk of a choice of solution by looking at its correlation with other highprobability solutions. If we treat the choice of solution as an alternative candidate for a 

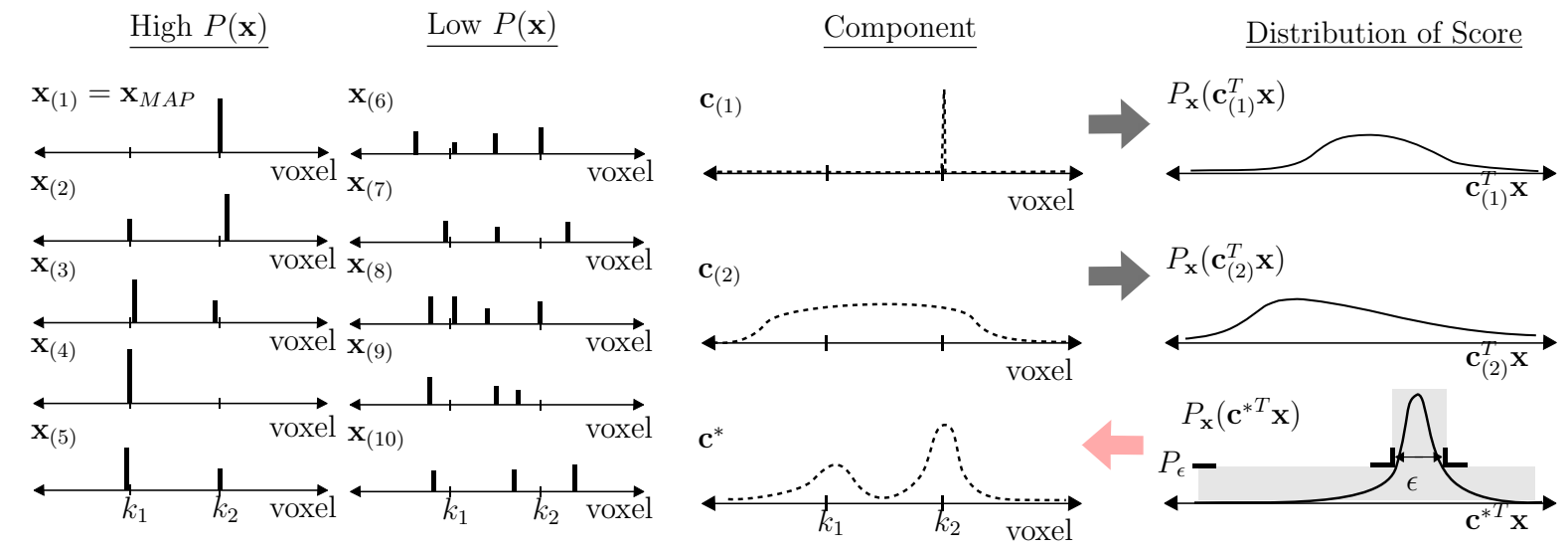

Figure 3: Example giving several solutions $\mathbf{x}$ with higher and lower $P(\mathbf{x})$, candidate components, and the marginal probability distribution of feature values; The broader the spread of the values for a given feature, the more ambiguous the feature. The design problem of calculating $\mathbf{c}$ based on constraints $\epsilon$ and $P_{\epsilon}$ (which is based on $\alpha_{1}$ and $\alpha_{2}$ ), amounts to finding a component who's value has a controlled statistical spread.

component, we may equivalently evaluate this risk by examining the marginal distribution of the score computed by this component. In Fig. 3 we depict multiple options for components; $\mathbf{c}_{(1)}$ is the MAP solution, while $\mathbf{c}_{(2)}$ calculates the an average over a large region. Fig. 3 also gives the distributions for the scores computed by these components (i.e., the range of correlations with potential solutions), where we see that these two result in broad distributions. Such broad distributions imply a wide possible variation in correlation with the different possible solutions, meaning these components are not very unambiguous, and hence are poor representatives of the set of high-probability solutions. Our strategy is depicted in Fig. 3 by the distribution for $P\left(\mathbf{c}^{* T} \mathbf{x}\right)$, where we set desired limits on the spread of the correlations, and use optimization to choose an optimal $\mathbf{c}^{*}$ which fulfills these requirements. We can implement this strategy, therefore, using the conditions we derived in the previous section, recalling the relationship between the constraint sets and the distributions of Eq. (4).

Further, in order to choose the unambiguous component which is most useful for identifying properties of the mechanism, not only do we seek a component which is unambiguous, we also wish for the correlation with potential solutions to be maximized. To motivate this approach, consider the following robust strategy. Suppose instead of seeking an unambigu- 
ous component, we simply sought a component which had high correlations with all possible $\mathbf{x} \in S_{D R}$ using the following minimax strategy,

$$
\mathbf{x}^{*}=\arg \max _{\|\mathbf{c}\|=1} \min _{\mathbf{x} \in S_{D R}} \mathbf{c}^{T} \mathbf{x}
$$

Here we seek the c for which the worst-possible correlation with high-probability solutions $\mathbf{x} \in S_{D R}$ is maximized. If we exchange the maximization and minimization to get $\min _{\mathbf{x} \in S_{D R}} \max _{\|\mathbf{c}\|=1} \mathbf{c}^{T} \mathbf{x}$, then we can analytically solve the inner maximization to get $\min _{\mathbf{x} \in S_{D R}}\|\mathbf{x}\|_{2}$, which is a variation on the elastic net of Eq. (6). So the elastic net can be viewed as a robust strategy to choose a vector $\mathbf{c}$ which maximizes the worst-case correlation with possible mechanisms, defined by $S_{D R}$.

For an unambiguous component, the correlations $\mathbf{c}^{T} \mathbf{x}$ will be approximately equal for all $\mathbf{x} \in S_{D R}$ by design. Therefore, we can simply choose an unambiguous component with maximum correlation using the following optimization (which we will call the UMAX problem, for Unambiguous Maximum correlation),

$$
\begin{gathered}
\mathbf{c}_{U M A X}=\arg \max _{\mathbf{c}, \mathbf{x}} \mathbf{c}^{T} \hat{\mathbf{x}} \\
\mathbf{c} \in R_{S} \\
\|\mathbf{c}\| \leq 1 .
\end{gathered}
$$

where $\hat{\mathbf{x}}$ is any solution in $S_{D R}$, such as the LASSO solution $\mathbf{x}_{1}$. We also relaxed the unit length constraint to an inequality constraint $\|\mathbf{c}\| \leq 1$, making the optimization convex. In the extreme case where $S_{D R}$ is small and only contains close approximations to $\mathbf{x}_{1}$, the optimal $\mathbf{c}$ will converge to $\mathbf{x}_{1}$.

Optimization also provides an opportunity to impose additional properties on the component vector c. In particular, we may independently impose sparsity of our component (not to be confused with the sparsity prior which applies to the mechanism). In Eq. (16), we trade off a degree of sparsity on $\mathbf{c}$ and correlation with the mechanism, based on a choice 
of regularization parameter $\eta$.

$$
\begin{gathered}
\mathbf{c}_{\eta}=\quad \arg \max _{\substack{\mathbf{c}, \mathbf{y}, \mathbf{y}^{\prime}, \lambda_{1}, \lambda_{1}^{\prime}, \lambda_{2}, \lambda_{2}^{\prime}}}\left\{\mathbf{c}^{T} \mathbf{x}_{0}-\eta\|\mathbf{c}\|_{1}\right\} \\
\mathbf{b}^{T}\left(\mathbf{y}+\mathbf{y}^{\prime}\right)+\alpha_{1}\left(\lambda_{1}+\lambda_{1}^{\prime}\right)+\alpha_{2}\left(\lambda_{2}+\lambda_{2}^{\prime}\right) \leq \epsilon \\
\left\|\mathbf{A}^{T} \mathbf{y}-\mathbf{c}\right\|_{\infty} \leq \lambda_{1} \\
\left\|\mathbf{A}^{T} \mathbf{y}^{\prime}+\mathbf{c}\right\|_{\infty} \leq \lambda_{1}^{\prime} \\
\|\mathbf{y}\|_{2} \leq \lambda_{2} \\
\left\|\mathbf{y}^{\prime}\right\|_{2} \leq \lambda_{2}^{\prime} \\
\|\mathbf{c}\|_{2} \leq 1 .
\end{gathered}
$$

For comparison, the following provides a similar optimization without the imposition of prior knowledge,

$$
\begin{gathered}
\mathbf{c}_{E C}^{*}=\arg \max _{\mathbf{c}, \mathbf{y}}\left\{\mathbf{c}^{T} \mathbf{x}_{0}-\eta\|\mathbf{c}\|_{1}\right\} \\
\mathbf{A}^{T} \mathbf{y}=\mathbf{c} \\
\|\mathbf{c}\|_{2} \leq 1 .
\end{gathered}
$$

This component is simply the best choice according to our heuristic which can be found within the rowspace. Techniques which select combinations of principal components such as sparse PCA or supervised PCA, essentially form a variation of Eq. (17).

\section{Results}

In this section we will demonstrate the method, first with simulations which illustrate the ability to control the variance of the component, then with real data where we compare the method to LASSO and elastic net for feature selection.

\subsection{Simulation}

First, we performed a simulation which demonstrated the robustness of the unambiguous component (i.e., the limited spread of its correlation with all other potential mechanisms). For this example, the minimum $\ell_{1}$-norm (Basis Pursuit [8]) solution is itself not unique. To explore this, we use this solution set as $S_{D R}$, so $\alpha_{2}=0$, and $\alpha_{1}$ computed from the 
Basis Pursuit solution, $\alpha_{1}=\min _{\mathbf{x}}\|\mathbf{x}\|_{1}$ subject to $\mathbf{A x}=\mathbf{b}$. The matrix $\mathbf{A}$ is $20 \times 100$ with binary random elements $A_{i j} \in\{-1,+1\}$. Such highly-structured matrices often fail to yield unique solutions for $\ell_{1}$-norm based techniques [12]. The true solution $\mathbf{x}_{\text {true }}$ is a sparse vector, plotted in Fig. 4, and $\mathbf{b}=\mathbf{A x}_{\text {true }}$. Fig. 4 also gives the Basis Pursuit solution, and a variety of other solutions that have the same $\ell_{1}$-norm (computed randomly), to demonstrate the non-uniqueness of the Basis Pursuit solution for this system.

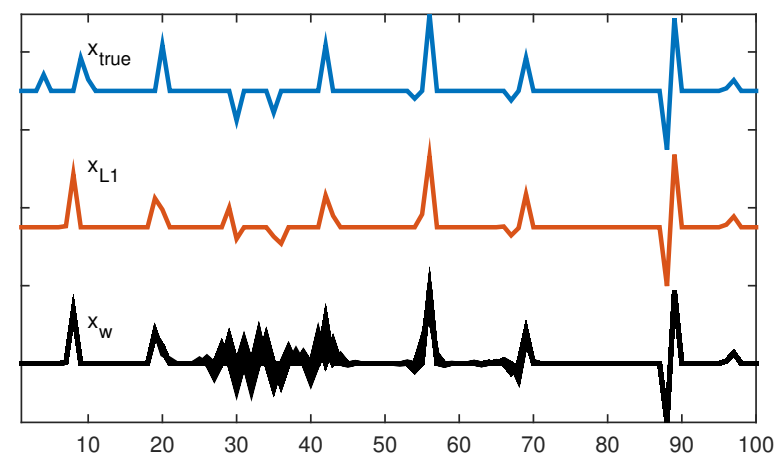

Figure 4: True $\mathbf{x}$, Basis Pursuit solution $\left(\mathbf{x}_{L 1}\right)$, and various other randomly-found solutions $\left(\mathbf{x}_{w}\right)$ plotted on top of each other, where $\left\|\mathbf{x}_{L 1}\right\|_{1}=\left\|\mathbf{x}_{w}\right\|_{1}$.

In Fig. 5 we give the components computed via Eq. (16) with $\eta=0.1\left(\mathbf{c}_{0.1}\right)$ and with $\eta=0\left(\mathbf{c}_{\max }\right)$. We used $\epsilon=0.01$ to allow for finite numerical precision. We also computed the analogous maximum component in the rowspace, $\mathbf{c}_{E C}$, using $\eta=0$ in Eq. (17).

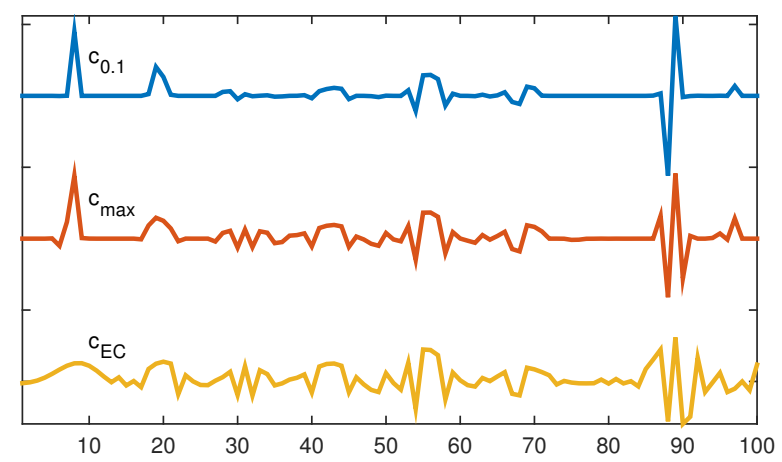

Figure 5: Different candidates for computing feature.

To demonstrate the robust behavior of the components, we used the optimization approach of Eq. (12) to measure the range of values each component $\mathbf{c}^{T} \mathbf{x}$ could take. We 
repeated this optimization over a range of relaxations of the prior knowledge, where we replaced $\alpha_{1}$ with $\alpha_{1}+\delta_{1}$ as in the following,

$$
\begin{gathered}
p\left(\delta_{1}\right)=\max _{\mathbf{x}, \mathbf{x}^{\prime}} \mathbf{c}^{T}\left(\mathbf{x}-\mathbf{x}^{\prime}\right) \\
\|\mathbf{x}\|_{1} \leq \alpha_{1}+\delta_{1} \\
\left\|\mathbf{x}^{\prime}\right\|_{1} \leq \alpha_{1}+\delta_{1} \\
\|\mathbf{A x}-\mathbf{b}\|_{2} \leq \alpha_{2} \\
\\
\left\|\mathbf{A} \mathbf{x}^{\prime}-\mathbf{b}\right\|_{2} \leq \alpha_{2}
\end{gathered}
$$

So when $\delta_{1}=0$, we have a test of the correlations with all possible solutions that have the same $\ell_{1}$-norm as the Basis Pursuit solution. In Fig. 6 we see that the unambiguous components we computed achieve $p \leq 0.01$ for $\delta_{1} \rightarrow 0$, as they were designed to, and as does the rowspace solution $\mathbf{c}_{E C}$. Once this prior knowledge is relaxed by increasing $\delta_{1}$, however, $\mathbf{c}_{\max }$ and $\mathbf{c}_{0.1}$ cease to yield unique values for $\mathbf{c}^{T} \mathbf{x}$. For comparison we also demonstrate

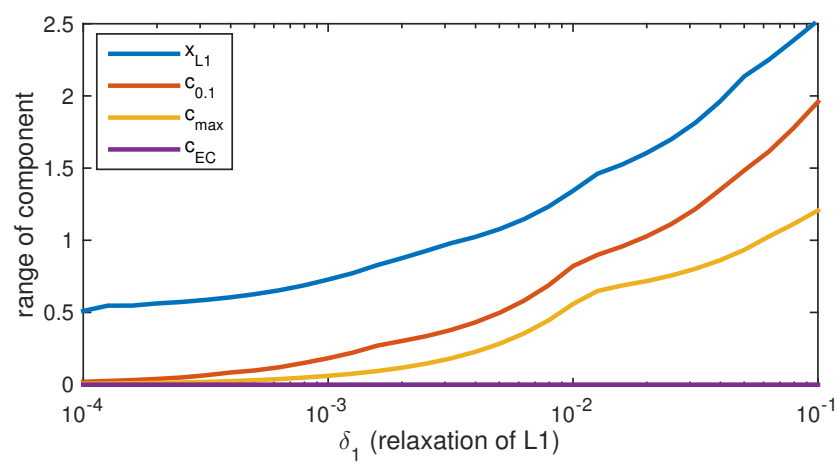

Figure 6: $p\left(\delta_{1}\right)$ computed via Eq. (12) for component values $\mathbf{c}^{T} \mathbf{x}$ over a relaxed version of $S_{D R}$ based on using $\alpha_{1}+\delta_{1}$, legend entries are arranged in same order as plot traces, from top to bottom

the correlation with the Basis Pursuit solution itself, $\mathbf{x}_{L 1}$. We see that it always results in a higher range $p$ for the ambiguity, which demonstrates that $\mathbf{x}_{L 1}$ is a poorer choice for a component, as its correlation varies significantly more over the possible predictors.

Next we repeated the example but with more relaxed choices for $S_{D R}$ in the component design, achieved by choosing larger values for $\alpha_{1}$. We also used different values for $\epsilon$, the constraint on allowable variation in correlations. The results for three different combinations are given in Fig. 7 for three different combinations of $\epsilon$ and $\alpha_{1}$. For each example, we chose 
a new pair of both $\alpha_{1}^{\prime}$ and $\epsilon$, where $\alpha_{1}^{\prime}$ is relaxed by a given amount versus the Basis Pursuit optimal $\alpha_{1}$ used in the previous example. We again plotted the performance of these components over increasingly-relaxed versions of $S_{D R}$, where we see the features are bounded by the chosen amount of relaxation within the limit given by the chosen $\epsilon$. Further

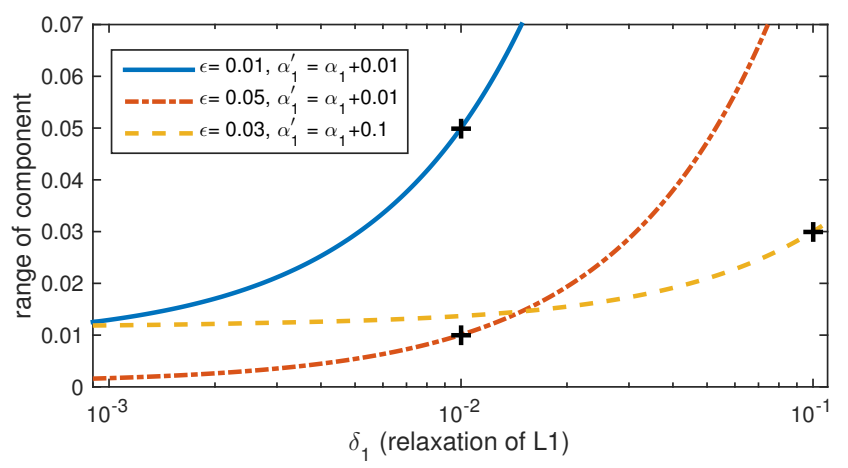

Figure 7: $p\left(\delta_{1}\right)$ computed via Eq. (12) for component values $\mathbf{c}^{T} \mathbf{x}$ over a relaxed set using three different combinations of $\delta_{1}$ and $\epsilon$.

we see that the choice of $\epsilon$ and $\alpha_{1}$ can control the robustness of the feature beyond the constraint set, as the range of correlations smoothly increase with a curve which depends on the conditions used. This suggests that by carefully designing $S_{D R}$, we can calculate components which robustly compute the information we seek.

\subsection{Biomarker Identification from Real fMRI Data}

Next we will use the calculated components for finding regions which most accurately classify diseases. We consider a dataset consisting of functional MRI images for a number of subjects which are labeled as cases or controls. We will first use cross-validation to optimize a LASSO regression estimate, in order to find a MAP estimate with the best possible accuracy in differentiating cases versus controls, and this will set the standard we wish to improve on. Then with this as a starting point, we will relax the prior knowledge a controlled amount and use cross-validation to test for improvement in terms of accuracy with unambiguous components. Accuracy is directly computed using the sign of the component score $\mathbf{c}^{T} \mathbf{a}_{i}$ versus the true class, where $\mathbf{a}_{i}$ is an image in the test set. The goal is to take advantage of 
the behavior we saw in the simulations as the prior is relaxed, to improve robustness while at the same time maximizing the ability to discriminate cases versus controls.

We used the data from a study comparing psychiatric patients to controls during an auditory sensorimotor task, conducted by The Mind Clinical Imaging Consortium (MCIC) [15]. The study included 208 participants, 92 of whom were diagnosed with schizophrenia, schizophreniform or schizoaffective disorder; the remaining 116 were healthy controls. The fMRI data were preprocessed using the statistical parametric mapping (SPM) software [25]; contrast images associated with the auditory stimuli form the data samples we use. Further details of the data collection and preprocessing can be found in our previous study [7].

Fig. 8 provides projections of the components or predictors calculated with several different methods. For PCA, Logistic LASSO, and Elastic Net, we used Matlab [16]. The sparse principal component was calculated using the SpaSM toolbox [28], with a setting of 100 nonzero voxels. The regularization parameters for all methods were chosen using 10-fold cross-validation. For UMAX (the proposed method) we started from the norms of the LASSO solution, then performed a line search for the optimal choice of relaxation, and picked the result with highest accuracy.

Both the first principal component and the sparse principal component took largest values in the occipital lobe, unlike the supervised methods, suggesting the signal variability was highest there, but not significantly related to the phenotype. The univariate correlation was highest in the precentral and postcentral gyrus, as noted in [7]. This was also evident in the LASSO, Logistic elastic, and UMAX results. The logistic elastic and UMAX result also included more of the neighboring sensory and motor association cortex, and UMAX in particular included a much higher weight to regions of the prefrontal cortex. In Fig. 9 we give the results of testing these components over relaxations of $S_{D R}$, suggesting how robust they are to the actual degree of sparsity of the true predictor (measured by relaxations of the $\ell_{1}$-norm). Again we see the controlled ambiguity of the UMAX method. Interestingly, we see that the sparse PCA component had higher ambiguity.

To compare the components' value as biomarkers or for inspiring further research into particular regions, we considered their accuracy at finding regions relevant to the disease. 

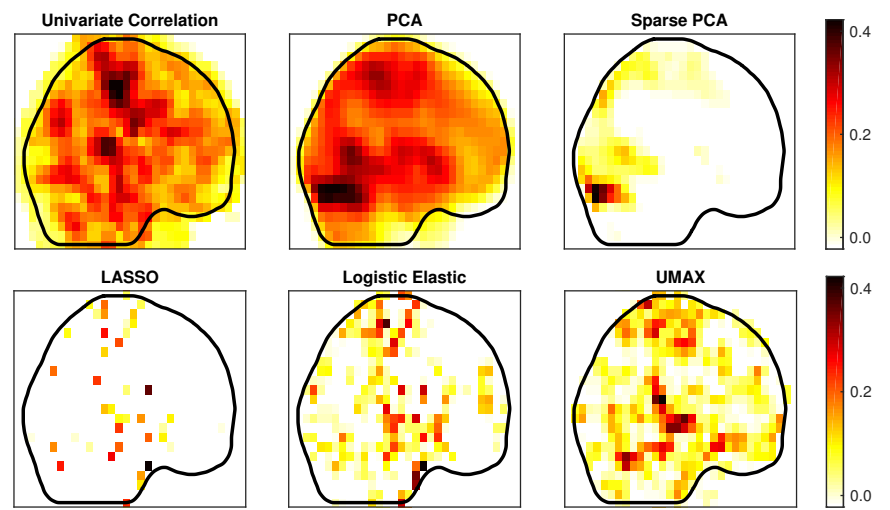

Figure 8: Projections of components calculated using: Pearson Correlation, PCA, Sparse PCA, LASSO, Logistic Elastic Net, and UMAX (proposed method).

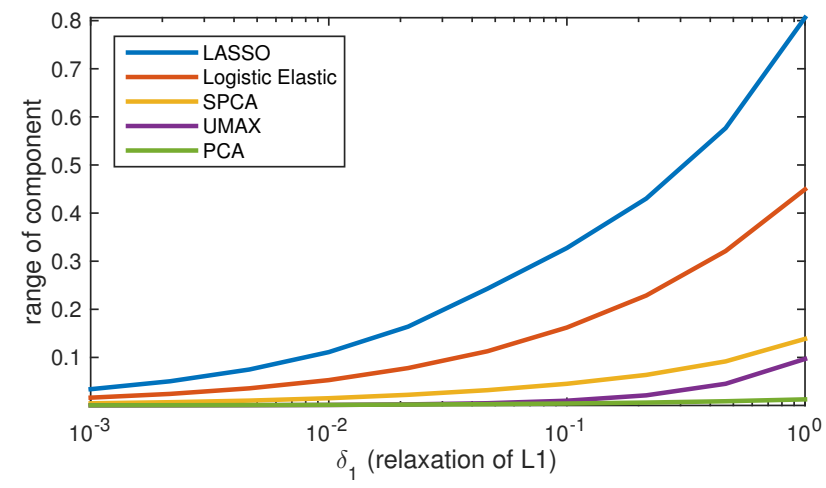

Figure 9: Component ranges for different elements versus sets of L1-regularized solutions; legend entries are arranged in same order as plot traces, from top to bottom.

Hence we tested the other features' classification performance directly by comparing the sign of the feature score $\mathbf{c}^{T} \mathbf{a}_{i}$ to the sign of $b_{i}$, where $\mathbf{a}_{i}$ is a sample in our test set, $\mathbf{c}$ is a feature tested, and $b_{i} \in\{+1,-1\}$ is the phenotype for the sample. In Table 1 we give the best accuracy (defined as the fraction of total test samples which were correctly classified, as used to determine the parameter values) and Pearson correlation achieved by each of the six features in 10-fold cross validation. As expected, we find that the PCA and sparse PCA components are not relevant to the disease. The LASSO solution performed fairly poorly and extensions such as elastic net (which allow a denser solution) and logistic elastic net, yielded minor improvements. Relaxation using the UMAX achieved a more significant improvement, both in terms of accuracy and correlation. We note that the overall accuracies 
Table 1: Methods and selectivity of different components compared to disease status.

\begin{tabular}{ccc} 
Method & Accuracy & Correlation \\
\hline \hline PCA & $48.6 \%$ & -0.08 \\
\hline Sparse PCA & $54.3 \%$ & 0.03 \\
\hline LASSO & $62.1 \%$ & 0.23 \\
\hline Elastic & $62.5 \%$ & 0.26 \\
\hline Logistic Elastic & $65.4 \%$ & 0.31 \\
\hline UMAX & $69.2 \%$ & 0.35 \\
\hline
\end{tabular}

achieved here are in line with meta-analyses [27], which generally suggest accuracies of 65 to 70 percent for samples of this size.

Next we identified the regions of interest (ROI) containing the signal for each of the components, using the Automated Anatomical Labeling (AAL) parcellation [31]. In Fig. 10 we give the number of common and different ROI for the three methods. We see that the elastic net essentially identifies a superset of the LASSO selections, while the proposed method only agrees with about two-thirds of each. Projections of the common and different

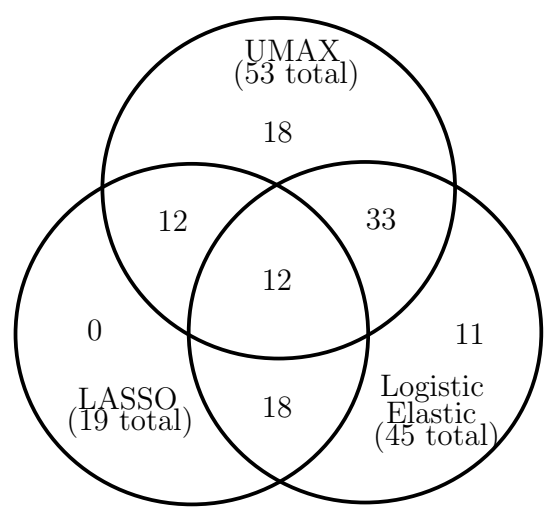

Figure 10: Intersections and differences in ROI containing the nonzero signal for the different components; the elastic net result is essentially a superset of LASSO, while the UMAX overlaps with roughly two thirds of each.

ROI (for a comparison of UMAX versus logistic elastic net) are provided in Fig. 11, and the names of ROI's are provided in the appendix. 

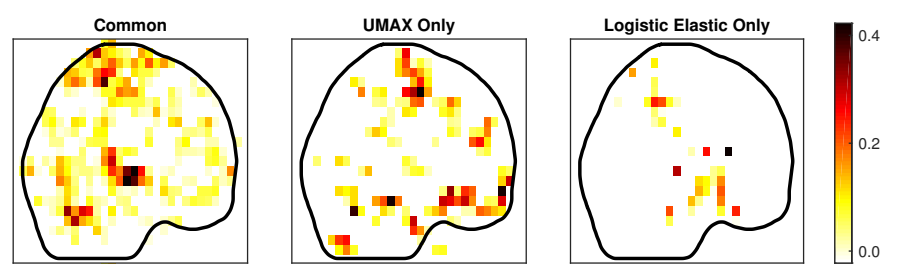

Figure 11: Projections of components contained in ROI's common to Logistic Elastic Net and UMAX estimate, and projections of those in only one result but not other.

\section{Discussion}

In this paper we provided a robust supervised framework for utilizing prior knowledge to find pertinent components of biological mechanisms. We demonstrated promising preliminary results using the technique for neuroimaging data, where we found that the choice of a maximum correlation component provided better accuracy as a classifier of case versus control. The use of the $\ell_{1}$-norm assumes a Laplacian prior, and therefore common significance testing approaches, which presume Gaussian statistics, are not applicable. Hence we used the best accuracy seen in cross-validation as the metric to determine performance. We found a limited degree of relaxation of the prior provides improvement, as the simulations suggested we might, particularly given the fact that the prior appeared to be of limited effectiveness. As the scope of this paper is the introduction of a broadly-applicable method, where many possible forms of prior may be used, we did not delve more deeply into the more specific issues of $\ell_{1}$-regression and associated statistical issues. In future work we intend to focus on both the development of more specialized priors, as well as associated significance testing approaches.

Potential difficulties of the method relate to the computational complexity, both in terms of algorithmic complexity, as well as the number of parameters to be chosen. We can view the latter (indeed all variables except c) as internal variables chosen by the algorithm itself, for example via cross-validation as in the previous section. The advantages of the method result from its rigorous formulation as a combination of dimensionality reduction with prior knowledge. We saw that this provides significant advantage for extremely noisy and underdetermined problems such as classification with neuroimaging data. 


\section{Appendix: ROI Listing}

Table 2: ROI common to UMAX and Logistic Elastic Net.

\begin{tabular}{ccc}
\hline \hline Postcentral_R & Temporal_Sup_R & Postcentral_L \\
Frontal_Mid_L & Temporal_Sup_L & Precentral_L \\
Cingulum_Mid_L & Temporal_Mid_L & Paracentral_Lobule_L \\
Cuneus_L & Precuneus_L & Precuneus_R \\
Cerebelum_6_L & Frontal_Sup_R & Parietal_Sup_R \\
Calcarine_L & Cingulum_Post_L & Cerebelum_6_R \\
Frontal_Sup_Medial_L & Supp_Motor_Area_R & Cerebelum_Crus1_L \\
Frontal_Inf_Tri_R & Frontal_Med_Orb_R & Temporal_Pole_Sup_L \\
Cerebelum_4_5_R & Fusiform_R & Lingual_L \\
Occipital_Mid_L & Paracentral_Lobule_R & Temporal_Mid_R \\
Lingual_R & Rolandic_Oper_L & Caudate_R \\
\hline
\end{tabular}


Table 3: ROI in either UMAX or Logistic Elastic Net which is not common to other.

\begin{tabular}{cc} 
UMAX Only & Logistic Elastic Only \\
\hline \hline Frontal_Mid_R & Parietal_Inf_L \\
Precentral_R & Olfactory_L \\
Temporal_Inf_R & Insula_R \\
Frontal_Med_Orb_L & Caudate_L \\
Cerebelum_Crus2_L & Parietal_Sup_L \\
ParaHippocampal_R & Putamen_L \\
Supp_Motor_Area_L & Thalamus_R \\
Cingulum_Ant_L & Cerebelum_3_R \\
Olfactory_R & SupraMarginal_R \\
Parietal_Inf_R & Rolandic_Oper_R \\
Frontal_Sup_Orb_L & Temporal_Pole_Sup_R \\
Fusiform_L & Hippocampus_L \\
Frontal_Sup_Orb_R & \\
Cerebelum_4_5_L & \\
SupraMarginal_L & \\
Calcarine_R & \\
Frontal_Inf_Orb_L & \\
Cingulum_Mid_R & \\
Rectus_R & \\
Insula_L & \\
\hline
\end{tabular}

\section{ACKNOWLEDGMENT}

The authors wish to thank the NIH (R01 GM109068, R01 MH104680, R01 MH107354) and NSF (1539067) for their partial support.

\section{References}

[1] Pasquale L. De Angelis, Panos M. Pardalos, and Gerardo Toraldo. Quadratic Programming with Box Constraints. In Immanuel M. Bomze, Tibor Csendes, Reiner Horst, and Panos M. Pardalos, editors, Developments in Global Optimization, number 18 in Nonconvex Optimization and Its Applications, pages 73-93. Springer US, 1997. 
[2] Eric Bair, Trevor Hastie, Debashis Paul, and Robert Tibshirani. Prediction by Supervised Principal Components. Journal of the American Statistical Association, 101(473):119-137, March 2006.

[3] Elnaz Barshan, Ali Ghodsi, Zohreh Azimifar, and Mansoor Zolghadri Jahromi. Supervised principal component analysis: Visualization, classification and regression on subspaces and submanifolds. Pattern Recognition, 44(7):1357-1371, July 2011.

[4] Stephen P. Boyd and Lieven Vandenberghe. Convex Optimization. Cambridge University Press, March 2004.

[5] Stuart Buck. Solving reproducibility. Science, 348(6242):1403-1403, June 2015.

[6] Hongbao Cao, Junbo Duan, Dongdong Lin, Yin Yao Shugart, Vince Calhoun, and Yu-Ping Wang. Sparse representation based biomarker selection for schizophrenia with integrated analysis of fMRI and SNPs. NeuroImage, 102 Pt 1:220-228, November 2014.

[7] Jiayu Chen, Vince D. Calhoun, Godfrey D. Pearlson, Stefan Ehrlich, Jessica A. Turner, Beng-Choon Ho, Thomas H. Wassink, Andrew M. Michael, and Jingyu Liu. Multifaceted genomic risk for brain function in schizophrenia. NeuroImage, 61(4):866-875, July 2012.

[8] Scott Shaobing Chen, David L. Donoho, and Michael A. Saunders. Atomic Decomposition by Basis Pursuit. SIAM Review, 43(1):129-159, January 2001.

[9] Carlton Chu, Ai-Ling Hsu, Kun-Hsien Chou, Peter Bandettini, and ChingPo Lin. Does feature selection improve classification accuracy? Impact of sample size and feature selection on classification using anatomical magnetic resonance images. NeuroImage, 60(1):59-70, March 2012.

[10] Keith Dillon and Yeshaiahu Fainman. Bounding pixels in computational imaging. Applied Optics, 52(10):D55-D63, April 2013.

[11] Keith Dillon and Yeshaiahu Fainman. Element-wise uniqueness, prior knowledge, and data-dependent resolution. Signal, Image and Video Processing, pages 1-8, April 2016.

[12] Keith Dillon and Yu-Ping Wang. Imposing uniqueness to achieve sparsity. Signal Processing, 123:1-8, June 2016.

[13] George H. Dunteman. Principal Components Analysis. SAGE, May 1989.

[14] Philip E. Gill, Walter Murray, and Margaret H. Wright. Numerical linear algebra and optimization. Addison-Wesley Pub. Co., Advanced Book Program, 1991.

[15] Randy L. Gollub, Jody M. Shoemaker, Margaret D. King, Tonya White, Stefan Ehrlich, Scott R. Sponheim, Vincent P. Clark, Jessica A. Turner, Bryon A. Mueller, Vince Magnotta, Daniel OLeary, Beng C. Ho, Stefan Brauns, Dara S. Manoach, Larry Seidman, Juan R. Bustillo, John Lauriello, Jeremy Bockholt, Kelvin O. Lim, Bruce R. Rosen, S. Charles Schulz, Vince D. Calhoun, and Nancy C. Andreasen. The MCIC collection: a shared repository of multi-modal, multi-site brain image data from a clinical investigation of schizophrenia. Neuroinformatics, 11(3):367-388, July 2013. 
[16] Andrew Grace. MATLAB optimization Toolbox. The MathWorks Inc., Natick, USA, 1992.

[17] Isabelle Guyon and Andr Elisseeff. An Introduction to Variable and Feature Selection. J. Mach. Learn. Res., 3:1157-1182, March 2003.

[18] Matthieu Kowalski. Sparse regression using mixed norms. Applied and Computational Harmonic Analysis, 27(3):303-324, November 2009.

[19] JohnH. Krystal and MatthewW. State. Psychiatric Disorders: Diagnosis to Therapy. Cell, 157(1):201214, March 2014

[20] Steven Lemm, Benjamin Blankertz, Thorsten Dickhaus, and Klaus-Robert Mller. Introduction to machine learning for brain imaging. NeuroImage, 56(2):387-399, May 2011.

[21] Dongdong Lin, Hongbao Cao, Vince D. Calhoun, and Yu-Ping Wang. Sparse models for correlative and integrative analysis of imaging and genetic data. Journal of Neuroscience Methods, 237:69-78, November 2014.

[22] Shuangge Ma and Ying Dai. Principal component analysis based methods in bioinformatics studies. Briefings in Bioinformatics, 12(6):714-722, November 2011.

[23] George A. Milliken and Dallas E. Johnson. Analysis of Messy Data Volume 1: Designed Experiments, Second Edition. CRC Press, March 2009.

[24] Graziella Orr, William Pettersson-Yeo, Andre F. Marquand, Giuseppe Sartori, and Andrea Mechelli. Using Support Vector Machine to identify imaging biomarkers of neurological and psychiatric disease: A critical review. Neuroscience \&3 Biobehavioral Reviews, 36(4):1140-1152, April 2012.

[25] William D. Penny, Karl J. Friston, John T. Ashburner, Stefan J. Kiebel, and Thomas E. Nichols. Statistical Parametric Mapping: The Analysis of Functional Brain Images: The Analysis of Functional Brain Images. Academic Press, April 2011.

[26] C. Radhakrishna Rao and Helge Toutenburg. Linear Models: Least Squares and Alternatives. Springer Science \& Business Media, June 2013.

[27] Hugo G. Schnack and Ren S. Kahn. Detecting Neuroimaging Biomarkers for Psychiatric Disorders: Sample Size Matters. Neuroimaging and Stimulation, page 50, 2016.

[28] Karl Sjstrand, Line Harder Clemmensen, Rasmus Larsen, and Bjarne Ersbll. Spasm: A matlab toolbox for sparse statistical modeling. Journal of Statistical Software Accepted for publication, 2012.

[29] Robert Tibshirani. Regression shrinkage and selection via the lasso. Journal of the Royal Statistical Society. Series B (Methodological), pages 267-288, 1996.

[30] Ryan J. Tibshirani. The lasso problem and uniqueness. Electronic Journal of Statistics, 7:1456-1490, 2013.

[31] N. Tzourio-Mazoyer, B. Landeau, D. Papathanassiou, F. Crivello, O. Etard, N. Delcroix, B. Mazoyer, and M. Joliot. Automated anatomical labeling of activations in SPM using a macroscopic anatomical 
parcellation of the MNI MRI single-subject brain. NeuroImage, 15(1):273-289, January 2002.

[32] Ming Yuan and Yi Lin. Model selection and estimation in regression with grouped variables. Journal of the Royal Statistical Society: Series B (Statistical Methodology), 68(1):49-67, February 2006.

[33] Hui Zhang, Wotao Yin, and Lizhi Cheng. Necessary and Sufficient Conditions of Solution Uniqueness in 1-Norm Minimization. Journal of Optimization Theory and Applications, 164(1):109-122, August 2014.

[34] Hui Zou and Trevor Hastie. Regularization and variable selection via the Elastic Net. Journal of the Royal Statistical Society, Series B, 67:301-320, 2005.

[35] Hui Zou, Trevor Hastie, and Robert Tibshirani. Sparse Principal Component Analysis. Journal of Computational and Graphical Statistics, 15(2):265-286, June 2006. 